\title{
Leray's plane steady state solutions are nontrivial
}

\author{
Mikhail Korobkov, Konstantin Pileckas, and Remigio Russo
}

November 27, 2019

\begin{abstract}
Affiliations: School of Mathematical Sciences, Fudan University, Shanghai 200433, China; and Voronezh State University, Voronezh 394018, Russia; e-mail:korob@math.nsc.ru

Institute of Applied Mathematics, Vilnius University, Naugarduko Str., 24, Vilnius, 03225 Lithuania;

e-mail: konstantinas.pileckas@mif.vu.lt

Dipartimento di Matematica e Fisica Università degli studi della Campania "Luigi Vanvitelli," viale Lincoln 5, 81100, Caserta, Italy;

e-mail: remigio.russo@unicampania.it
\end{abstract}

Acknowledgment. M. Korobkov was partially supported by the Ministry of Education and Science of the Russian Federation (Grant 14.Z50.31.0037).

The research of K. Pileckas was funded by the grant No. S-MIP-17-68 from the Research Council of Lithuania.

\begin{abstract}
We study solutions to the obstacle problem for the stationary NavierStokes system in a two dimensional exterior domain (flow past a prescribed body). We prove that the classical Leray solution to this problem is always nontrivial. No additional condition (on symmetry or smallness, etc.) is assumed. This is a complete extension of a classical result of C.J. Amick (Acta Math. 1988) where nontriviality was proved under symmetry assumption.
\end{abstract}

2010 MSC: Primary 76D05, 35Q30; Secondary 31B10, 76D03;

Key words: stationary Stokes and Navier Stokes equations, two-dimensional exterior domains, asymptotic behavior 


\section{Introduction}

Let $\Omega$ be an exterior domain in $\mathbb{R}^{2}$ with compact boundary $\partial \Omega=\bigcup_{i=1}^{N} \Gamma_{i}$, where $\Gamma_{i}$ are smooth disjoint curves, homeomorphic to the circle. In particular, $\Omega \supset$ $\mathbb{R}^{2} \backslash B$, where $B$ is the disk of radius $R_{0}$ centered at the origin with $\partial \Omega \subset B$.

One of the most difficult and still open problem in the theory of the stationary Navier-Stokes equations, initiated by J. Leray in the famous paper of 1933 [12], concerns the existence of a solution to the flow around an obstacle (see also [5]):

$$
\left\{\begin{aligned}
-\nu \Delta \mathbf{u}+(\mathbf{u} \cdot \nabla) \mathbf{u}+\nabla p & =\mathbf{0} & & \text { in } \Omega \\
\operatorname{div} \mathbf{u} & =0 & & \text { in } \Omega \\
\mathbf{u} & =\mathbf{0} & & \text { on } \partial \Omega \\
\mathbf{u}(z) & \rightarrow \mathbf{u}_{0} & & \text { as }|z| \rightarrow \infty
\end{aligned}\right.
$$

where $\mathbf{u}$ and $p$ are the unknown velocity and pressure fields, $\nu$ denotes the kinematical viscosity coefficient, and $\mathbf{u}_{0} \in \mathbb{R}^{2}$ is a nonzero constant vector (prescribed velocity at infinity).

Leray suggested [12] the following elegant approach to this problem which was called method of "invading domains". Denoting by $\mathbf{u}_{k}$ the solution to the problem

$$
\left\{\begin{array}{rlrl}
-\nu \Delta \mathbf{u}_{k}+\left(\mathbf{u}_{k} \cdot \nabla\right) \mathbf{u}_{k}+\nabla p_{k}=\mathbf{0} & & \text { in } \Omega_{k}, \\
\operatorname{div} \mathbf{u}_{k}=0 & & \text { in } \Omega_{k}, \\
\mathbf{u}_{k}=\mathbf{0} & & \text { on } \partial \Omega, \\
\mathbf{u}_{k} & =\mathbf{u}_{0} & & \text { for }|z|=R_{k} .
\end{array}\right.
$$

on the intersection $\Omega_{k}$ of $\Omega$ with the disk $B_{R_{k}}$ of radius $R_{k} \geq k\left(\gg R_{0}\right)$, whose existence he proved before, Leray showed that the sequence $\mathbf{u}_{k}$ satisfies the estimate $\int_{\Omega}\left|\nabla \mathbf{u}_{k}\right|^{2} \leq c$ for some positive constant $c$ independent of $k$. Hence, he observed that it is possible to extract a subsequence $\mathbf{u}_{k_{n}}$ which weakly converges to a solution $\mathbf{u}_{L}$ of problem (1.1) $1,2,3$ with $\int_{\Omega}\left|\nabla \mathbf{u}_{L}\right|^{2}<+\infty$. This solution was later called Leray's solution (see, e.g., [1]).

An arbitrary solution $\mathbf{u}$ to the Navier-Stokes equations

$$
\left\{\begin{aligned}
-\nu \Delta \mathbf{u}+(\mathbf{u} \cdot \nabla) \mathbf{u}+\nabla p & =\mathbf{0} & & \text { in } \Omega \\
\operatorname{div} \mathbf{u} & =0 & & \text { in } \Omega .
\end{aligned}\right.
$$

having the finite Dirichlet integral

$$
\int_{\Omega}|\nabla \mathbf{u}|^{2}<+\infty,
$$


is called today $D$-solution [5]. As is well known (e.g., [11]), such solutions are real-analytic in $\Omega$.

As far as condition $(1.1)_{4}$ is concerned, Leray limited himself to observe that, while in three dimensional problem (1.4) it is sufficient to guarantee the attainability of the limit $\mathbf{u}_{0}$ at infinity (at least in a mean square sense) as a consequence of the inequality $\left\|r^{-1}\left(\mathbf{u}-\mathbf{u}_{0}\right)\right\|_{L^{2}(\Omega)} \leq 4\|\nabla \mathbf{u}\|_{L^{2}(\Omega)}$, in the two dimension case the corresponding inequality $\left\|(r \log r)^{-1}\left(\mathbf{u}-\mathbf{u}_{0}\right)\right\|_{L^{2}(\Omega)} \leq c\|\nabla \mathbf{u}\|_{L^{2}(\Omega)}$ does not imply any type of convergence. Leray concluded that one should not be surprised of this phenomenon, in view of the Stokes paradox, i.e., the system obtained from (1.1) removing the nonlinear term, namely,

$$
\left\{\begin{aligned}
-\nu \Delta \mathbf{u}+\nabla p & =\mathbf{0} & & \text { in } \Omega \\
\operatorname{div} \mathbf{u} & =0 & & \text { in } \Omega \\
\mathbf{u} & =\mathbf{0} & & \text { on } \partial \Omega, \\
\mathbf{u}(z) & \rightarrow \mathbf{u}_{0} & & \text { as }|z| \rightarrow \infty
\end{aligned}\right.
$$

does not admit a solution (see, e.g., [15]).

The problem of the asymptotic behaviour at infinity of Leray's solution $\left(\mathbf{u}_{L}, p_{L}\right)$ was tacked by D. Gilbarg \& H. Weinberger in 1974 [6]. They proved that $\mathbf{u}_{L}$ is bounded, there are a scalar $p_{0}$ and a constant vector $\mathbf{u}_{\infty}$ such that

$$
\lim _{|z| \rightarrow+\infty} p_{L}(z)=p_{0}
$$

(one can choose, say, $p_{0}=0$ ),

$$
\lim _{|z| \rightarrow+\infty} \int_{0}^{2 \pi}\left|\mathbf{u}_{L}(r, \theta)-\mathbf{u}_{\infty}\right|^{2} d \theta=0,
$$

and

$$
\begin{aligned}
& \omega(z)=o\left(r^{-3 / 4}\right), \\
& \int_{\Omega} r|\nabla \omega(z)|^{2}<\infty,
\end{aligned}
$$

where $r=|z|$ and

$$
\omega=\partial_{2} u_{L 1}-\partial_{1} u_{L 2}
$$

is the vorticity.

In 1988 C.J. Amick [1] proved that a $D$-solution to the problem of a flow around an obstacle (1.1) 1.2.3 has the following asymptotic properties:

(i) $\mathbf{u}$ is bounded and, as a consequence, it satisfies (1.7)-(1.8); 
(ii) the total head pressure $\Phi=p+\frac{1}{2}|\mathbf{u}|^{2}$ and the absolute value of the velocity $|\mathbf{u}|$ have the uniform limit at infinity, i.e.,

$$
|\mathbf{u}(r, \theta)| \rightarrow\left|\mathbf{u}_{\infty}\right| \quad \text { as } r \rightarrow \infty
$$

where $\mathbf{u}_{\infty}$ is the constant vector from the condition (1.7);

(iii) if $\partial \Omega$ is symmetric with respect to the $x_{1}$-axis, and $\mathbf{u}=\left(u_{1}, u_{2}\right)$ is also symmetric, i.e., if $u_{1}$ is even and $u_{2}$ is odd with respect to $x_{1}$, then $\mathbf{u}$ converges uniformly at infinity to a constant vector $\mu \mathbf{e}_{1}$, for some scalar $\mu$. Moreover, the Leray procedure yields a nontrivial (i.e., not identically zero) symmetric solution.

In the present paper we prove that in general case (without any additional symmetry assumptions) the Leray solution to the the problem of the flow around obstacle is always nontrivial.

Theorem 1.1. Let $\Omega$ be an exterior domain in $\mathbb{R}^{2}$ with smooth compact boundary, $\nu>0$ and $\mathbf{0} \neq \mathbf{u}_{0} \in \mathbb{R}^{2}$. Take a sequence $\mathbf{u}_{k}$ of solutions to system (1.2), and take further arbitrary weakly convergent subsequence $\mathbf{u}_{k_{n}} \rightarrow \mathbf{u}$. Then the limiting solution $\mathbf{u}$ to $(1.1)_{1,2,3}$ is nontrivial (i.e., $\mathbf{u}$ is not identically zero). In particular the Leray solution is nontrivial.

Moreover, we proved a kind of complementary result.

Theorem 1.2. Let $\Omega$ be an exterior domain in $\mathbb{R}^{2}$ with smooth compact boundary, $\nu>0$ and let $\mathbf{a} \in \mathbb{R}^{2}$ be a nonzero constant vector. Take a sequence $\mathbf{u}_{k}$ of solutions to the system

$$
\left\{\begin{aligned}
-\nu \Delta \mathbf{u}_{k}+\left(\mathbf{u}_{k} \cdot \nabla\right) \mathbf{u}_{k}+\nabla p_{k}=\mathbf{0} & \text { in } \Omega_{k}, \\
\operatorname{div} \mathbf{u}_{k}=0 & \text { in } \Omega_{k}, \\
\mathbf{u}_{k}=\mathbf{a} & \text { on } \partial \Omega, \\
\mathbf{u}_{k}=\mathbf{0} & \text { for }|z|=R_{k},
\end{aligned}\right.
$$

and take further arbitrary weakly convergent subsequence $\mathbf{u}_{k_{n}} \rightarrow \mathbf{u}$. Then the limiting solution $\mathbf{u}$ is nontrivial. In other words, the solution to the system

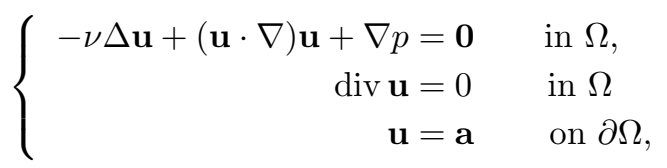

obtained by the Leray method is nontrivial, i.e., $\mathbf{u} \neq \mathbf{a}$. 
Note, that for linear case (e.g., for Stokes system (1.5)) the assertions of Theorems 1.1-1.2 are evidently equivalent. But of course it does not hold in general for nonlinear systems.

Recently [9], [10] we proved the following result for general $D$-solutions.

Theorem 1.3 ([10]). Let $\mathbf{u}$ be a D-solution to the Navier-Stokes system (1.3) in the exterior domain $\Omega \subset \mathbb{R}^{2}$. Then $\mathbf{u}$ converges uniformly at infinity, i.e.,

$$
\mathbf{u}(z) \rightarrow \mathbf{u}_{\infty} \quad \text { uniformly as }|z| \rightarrow \infty,
$$

where $\mathbf{u}_{\infty} \in \mathbb{R}^{2}$ is some constant vector.

By virtue of Theorem 1.3, for every Leray solution there is a constant vector $\mathbf{u}_{\infty} \in \mathbb{R}^{2}$ such that (1.11) holds. However, the desired equality $\mathbf{u}_{\infty}=\mathbf{u}_{0}$ is still an open question. We even do not know whether $\mathbf{u}_{\infty}$ is nonzero if $\mathbf{u}_{0} \neq \mathbf{0}$. So, the problem of the flow around an obstacle remains still open.

The same open problem exists in Theorem 1.2: we know that the corresponding Leray solution converges uniformly to some constant vector $\mathbf{u}_{\infty} \in \mathbb{R}^{2}$, but we were not able to prove the expected equality that $\mathbf{u}_{\infty}=\mathbf{0}$.

Some additional historical remarks. Note, that thirty years after Leray, H. Fujita [4] by means of different techniques proved the existence of a $D$-solution to $(1.1)_{1.2 .3}$. Due of a lack of a uniqueness theorem, the Leray and Fujita solutions are not comparable.

Recall also the amazing discovery of R. Finn and D.R. Smith in 1967 [3] of the existence of a solution to (1.1) for $\nu$ sufficiently large (or, equivalently, for $\mathbf{u}_{0}$ sufficiently small). Their approach is completely different from that of Leray. Nevertheless, their method does not allow to prove the existence of the solution to the problem

$$
\left\{\begin{aligned}
-\nu \Delta \mathbf{u}+(\mathbf{u} \cdot \nabla) \mathbf{u}+\nabla p & =\mathbf{0} & & \text { in } \Omega \\
\operatorname{div} \mathbf{u} & =0 & & \text { in } \Omega \\
\mathbf{u} & =\mathbf{a} & & \text { on } \partial \Omega \\
\mathbf{u}(z) & \rightarrow \mathbf{0} & & \text { as }|z| \rightarrow \infty
\end{aligned}\right.
$$

for a constant vector $\mathbf{a} \in \mathbb{R}^{2}$. This problem is quite open even for small vectors $\mathbf{a} \neq \mathbf{0}$ (the existence was proved for some nonconstant a under the assumption of symmetry with respect to both coordinate axes [13]). Even the reduced problem $\left(1.12_{1-3}\right)$ is open for general boundary value a (see, e.g., [14] for the case of small fluxes). More detailed survey of results concerning boundary value problems for stationary NS-system in plane exterior domains see, e.g., in [5] or in our recent papers [9], [10], [8]. 
Finally, let us describe shortly the main steps of the proof of Theorem 1.1 (for defineteness, take $\mathbf{u}_{0}=(1,0)$ and $\nu=1$ ). The main ideas are rather simple. By Amick criterion [1], the corresponding limiting Leray solution $\mathbf{u}$ is trivial if and only if the convergence

$$
\int_{\Omega_{k}}\left|\nabla \mathbf{u}_{k}\right|^{2} d x \rightarrow 0 \quad \text { as } k \rightarrow \infty
$$

holds for the sequence of solutions $\mathbf{u}_{k}$ to (1.2) in bounded domains $\Omega_{k}=$ $\Omega \cap B_{R_{k}}$. Suppose (1.13) to be fulfilled. Then the functions $\left(\mathbf{u}_{k}, p_{k}\right)$ and all its derivatives go to 0 uniformly on every bounded set; moreover, from [6] it follows that $\sup _{z \in \Omega^{\prime}}\left|p_{k}\right| \rightarrow 0$, where $\Omega_{k}^{\prime}=\Omega \cap B_{\frac{3}{4} R_{k}}$. It is well known, that the Bernoulli $z \in \Omega_{k}^{\prime}$

pressure $\Phi_{k}=p_{k}+\frac{1}{2}\left|\mathbf{u}_{k}\right|^{2}$ satisfies the maximum principle. Using these facts, it can be proved that the level lines $\Phi_{k}=t$ of the Bernoulli pressure are arranged as circles surrounding the origin; furthermore, the Bernoulli pressure approximately equal to zero near $\partial \Omega$, and it increases up to $\frac{1}{2}$ near the large circle of a radius $R_{k}$.

The following steps are crucial in our arguments:

1) The direction of the velocity vector $\mathbf{u}_{k}$ is under control of the Dirichlet integral (it was proved in the Gilbarg-Weinberger paper [7], see below lemma 2.4 for the exact formulation of the result);

2) The vorticity $\omega_{k}(z)$ does not change sign between two level lines of the Bernoulli pressure $\Phi_{k}$ (it is proved using the results of Amick [1]).

Using these important facts, we prove that for the velocity $\mathbf{u}_{k}$ the following representation formulas hold:

$$
\overline{\mathbf{u}}_{k}(r)=\left|\overline{\mathbf{u}}_{k}(r)\right|\left(\cos \varphi_{k}(r), \sin \varphi_{k}(r)\right),
$$

where $\overline{\mathbf{u}}_{k}(r)$ means the mean value of $\mathbf{u}_{k}$ over the circle of radius $r$ and

$$
\left|\varphi_{k}(r)\right| \leq \varepsilon_{k} \quad \text { for all sufficiently large } r \leq R_{k}
$$

with $\varepsilon_{k} \rightarrow 0$ as $k \rightarrow \infty$. Recall, that the gradient of the Bernoulli pressure satisfies the identity

$$
\nabla \Phi_{k}(z)=-\nabla^{\perp} \omega_{k}(z)+\omega_{k}(z) \cdot \mathbf{u}_{k}^{\perp}(z),
$$

where $\mathbf{u}_{k}^{\perp}:=\left(-u_{k}^{2}, u_{k}^{1}\right)$. The first term in (1.16) is negligible (since the integral $\int r\left|\nabla \omega_{k}\right|^{2}$ is small, see $\left.\left(1.8_{2}\right)\right)$. Using these facts we obtain the contradic$\Omega_{k}^{\prime}$

tion with the geometrical structure of the level lines of the Bernoulli pressure $\Phi_{k}$ described above and, as a consequence, the nontriviality of the Leray solution. 


\section{Notations and preliminaries}

By a domain we mean an open connected set. We use standard notations for Sobolev spaces $W^{k, q}(\Omega)$, where $k \in \mathbb{N}, q \in[1,+\infty]$. In our notation we do not distinguish function spaces for scalar and vector valued functions; it is clear from the context whether we use scalar or vector (or tensor) valued function spaces.

For $q \geq 1$ denote by $D^{k, q}(\Omega)$ the set of functions $f \in W_{\text {loc }}^{k, q}(\Omega)$ such that $\|f\|_{D^{k, q}(\Omega)}=\left\|\nabla^{k} f\right\|_{L^{q}(\Omega)}<\infty$.

We denote by $\mathcal{H}^{k}$ the $k$-dimensional Hausdorff measure, i.e., $\mathcal{H}^{k}(F)=$ $\lim _{t \rightarrow 0+} \mathcal{H}_{t}^{k}(F)$, where

$$
\mathcal{H}_{t}^{1}(F)=\left(\frac{\alpha_{k}}{2}\right)^{k} \inf \left\{\sum_{i=1}^{\infty}\left(\operatorname{diam} F_{i}\right)^{k}: \operatorname{diam} F_{i} \leq t, F \subset \bigcup_{i=1}^{\infty} F_{i}\right\}
$$

and $\alpha_{k}$ is a Lebesgue volume of the unit ball in $\mathbb{R}^{k}$.

In particular, for a curve $S$ the value $\mathcal{H}^{1}(S)$ coincides with its length, and for sets $E \subset \mathbb{R}^{2}$ the $\mathcal{H}^{2}(E)$ coincides with the usual Lebesgue measure in $\mathbb{R}^{2}$.

Also, for a curve $S$ by $\int_{S} f d s$ we denote the usual integral with respect to 1-dimensional Hausdorff measure (=length). Further, for a set $E \subset \mathbb{R}^{2}$ by $\int_{E} f(\xi) d \mathcal{H}^{2}$ we denote the integral with respect to the two-dimensional Lebesgue measure. For convenience, we will write simply

$$
\int_{E} r f \quad \text { instead of } \quad \int_{E}|\xi| f(\xi) d \mathcal{H}_{\xi}^{2} .
$$

Below we present some results concerning the behavior of $D$-functions.

Lemma 2.1. Let $f \in D^{1,2}(\Omega)$ and assume that

$$
\int_{D}|\nabla f|^{2}<\varepsilon^{2}
$$

for some $\varepsilon>0$ and for some ring $D=\left\{z \in \mathbb{R}^{2}: r_{1}<\left|z-z_{0}\right|<r_{2}\right\} \subset \Omega$. Then the estimate

$$
\left|\bar{f}\left(r_{2}\right)-\bar{f}\left(r_{1}\right)\right| \leq \varepsilon \sqrt{\ln \frac{r_{2}}{r_{1}}}
$$

holds, where $\bar{f}$ is the mean value of $f$ over the circle $S\left(z_{0}, r\right)$ :

$$
\bar{f}(r):=\frac{1}{2 \pi r} \int_{\left|z-z_{0}\right|=r} f(z) d s .
$$


Lemma 2.2. Fix a number $\beta \in(0,1)$. Let $f \in D^{1,2}(\Omega)$ and assume that

$$
\int_{D}|\nabla f|^{2}<\varepsilon^{2}
$$

for some $\varepsilon>0$ and for some ring $D=\left\{z \in \mathbb{R}^{2}: \beta R<\left|z-z_{0}\right|<R\right\} \subset \Omega$. Then there exists a number $r \in[\beta R, R]$ such that the estimate

$$
\sup _{\left|z-z_{0}\right|=r}|f(z)-\bar{f}(r)| \leq c_{\beta} \varepsilon
$$

holds, where the constant $c_{\beta}$ depends on $\beta$ only.

The proofs of above lemmas are standard, see, e.g., [7] for the proofs of similar results. Summarizing the results of these lemmas, we receive

Lemma 2.3. Under conditions of Lemma 2.2, there exists $r \in[\beta R, R]$ such that

$$
\sup _{\left|z-z_{0}\right|=r}|f(z)-\bar{f}(R)| \leq \tilde{c}_{\beta} \varepsilon
$$

The following result was proved in [7, Theorem 4, page 399]. It means, roughly speaking, that the direction of the velocity vector $\mathbf{u}$ satisfying the Navier-Stokes system is controlled by the Dirichlet integral.

Lemma 2.4 ([7]). Let $\mathbf{u}$ be a D-solution to the Navier-Stokes system (1.3) in the exterior domain $\Omega \subset \mathbb{R}^{2}$, and let $D=\left\{z \in \mathbb{R}^{2}: R_{1}<|z|<R_{2}\right\} \subset \Omega$ be some ring in $\Omega$. Denoted by $\overline{\mathbf{u}}(r)$ the mean value of $\mathbf{u}$ over the circle $S_{r}$ :

$$
\overline{\mathbf{u}}(r)=\frac{1}{2 \pi r} \int_{|\xi|=r} \mathbf{u}(\xi) d s
$$

and let $\varphi(r)$ be the argument of the complex number associated to the vector $\overline{\mathbf{u}}(r)=\left(\bar{u}_{1}(r), \bar{u}_{2}(r)\right)$, i.e., $\varphi(r)=\arg \left(\bar{u}_{1}(r)+i \bar{u}_{2}(r)\right)$. Assume also that

$$
|\overline{\mathbf{u}}(r)| \geq \sigma
$$

for some positive constant $\sigma>0$ and for all $r \in\left[R_{1}, R_{2}\right]$. Then the following estimate

$$
\sup _{R_{1}<\rho_{1} \leq \rho_{2} \leq R_{2}}\left|\varphi\left(\rho_{2}\right)-\varphi\left(\rho_{1}\right)\right| \leq \frac{1}{4 \pi \sigma^{2}} \int_{D}\left(\frac{1}{r}|\nabla \omega|+|\nabla \mathbf{u}|^{2}\right)
$$

holds. 
The following statement also follows from (2.5).

Corollary 2.1. Under the conditions of Lemma 2.4, the estimate

$$
\sup _{R_{1}<\rho_{1} \leq \rho_{2} \leq R_{2}}\left|\varphi\left(\rho_{2}\right)-\varphi\left(\rho_{1}\right)\right| \leq \frac{1}{2 \sigma^{2} R_{1}}+\frac{1}{4 \pi \sigma^{2}} \int_{D}\left(r|\nabla \omega|^{2}+|\nabla \mathbf{u}|^{2}\right)
$$

holds.

\section{Proof of the main Theorem 1.1.}

We prove Theorem 1.1 by getting a contradiction. Suppose the assumptions of Theorem 1.1 are fulfilled, but the statement is false, i.e., there exists an increasing sequence of radii $R_{k} \rightarrow+\infty$ and solutions $\mathbf{u}_{k}$ to the system (1.2) such that $\mathbf{u}_{k_{n}} \rightarrow \mathbf{u} \equiv \mathbf{0}$. By the result of Amick [1, Theorem 24, page 115], it is equivalent to the global convergence to zero of the Dirichlet integrals:

$$
\int_{\Omega_{k}}\left|\nabla \mathbf{u}_{k}\right|^{2} \rightarrow 0
$$

By classical regularity results for $D$-solutions to the Navier-Stokes system (e.g., [5]), the functions $\mathbf{u}_{k}$ and $p_{k}$ are $C^{\infty}$-smooth on the set $\bar{\Omega}_{k}$ and real analytical inside $\Omega_{k}$. Moreover, (3.1) implies in particular, that for every compact set $E \subset \bar{\Omega}$

$$
\sup _{x \in E}\left|\nabla^{j} \mathbf{u}_{k}(x)\right| \rightarrow 0 \quad \forall j=0,1,2, \ldots
$$

uniformly as $k \rightarrow \infty$, i.e., $\mathbf{u}_{k}$ and all its derivatives converges to zero as $k \rightarrow \infty$ uniformly on every compact set.

Without loss of generality we may assume that

$$
\nu=1 \quad \text { and } \quad \mathbf{u}_{0}=(1,0) .
$$

The proof consists of eight steps.

SteP 1. Denote $\Omega_{k}^{\prime}=\Omega \cap B\left(0, \frac{3}{4} R_{k}\right)$. By results of [6]-[7] (see the proofs of Lemmas 2.2 and 3.2 in [6]), the following estimate

$$
\int_{\Omega_{k} \cap B\left(0, \frac{3}{4} R_{k}\right)} r\left|\nabla \omega_{k}\right|^{2} \leq c \int_{\Omega_{k}}\left|\omega_{k}\right|^{2} \leq c \int_{\Omega_{k}}\left|\nabla \mathbf{u}_{k}\right|^{2}
$$


holds with the constant $c$ independent of $k$. Hence the assumption (3.1) yields

$$
\int_{\Omega_{k}^{\prime}} r\left|\nabla \omega_{k}\right|^{2} \rightarrow 0
$$

Moreover, from [6]-[7] it follows that (see [6, Lemma 2.4])

$$
\sup _{x \in \Omega_{k}^{\prime}}\left|\mathbf{u}_{k}(x)\right| \leq C .
$$

and (see the proofs of Lemmas 2.3-2.6 in [6] and, in particular, [6, formula (2.20)] )

$$
\sup _{x \in \Omega_{k}^{\prime}}\left|p_{k}(x)\right|=\varepsilon_{k} .
$$

Here and everywhere below the equality $a_{k}=\varepsilon_{k}$ means that the sequence $a_{k}$ tends to 0 as $k \rightarrow \infty$.

SteP 2. Denote

$$
R_{0 k}=\min \left\{r \geq R_{0}:\left|\overline{\mathbf{u}}_{k}(r)\right|=\left|f_{S_{r}} \mathbf{u}_{k} d s\right|=\frac{1}{5}\right\},
$$

where, as usual, $S_{r}=\left\{\xi \in \mathbb{R}^{2}:|\xi|=r\right\}$ is a circle. From (3.2) we have, in particular,

$$
R_{0 k} \rightarrow+\infty \quad \text { as } k \rightarrow \infty .
$$

Moreover, Lemma 2.1 applied to $\mathbf{u}_{k}$ and the identity $\overline{\mathbf{u}}_{k}\left(R_{k}\right)=(1,0)$ imply, by virtue of (3.1), that

$$
\frac{R_{k}}{R_{0 k}} \rightarrow+\infty \quad \text { as } k \rightarrow \infty .
$$

STEP 3. By construction (see (3.6), (3.7)),

$$
\max _{x \in S_{R_{0 k}}} \Phi_{k}(x)>\frac{1}{2}\left(\frac{1}{5}\right)^{2}+\varepsilon_{k}=\frac{1}{50}+\varepsilon_{k}>\frac{1}{60}>\max _{x \in \partial \Omega} \Phi_{k}(x),
$$

for sufficiently large $k$, where, recall, $\Phi_{k}=p_{k}+\frac{1}{2}\left|\mathbf{u}_{k}\right|^{2}$ is the Bernoulli pressure. It is well known, that $\Phi_{k}$ satisfies the identity

$$
\Delta \Phi_{k}=\omega_{k}^{2}+\nabla \Phi_{k} \cdot \mathbf{u}_{k},
$$

and thus, it satisfies the classical strong maximum principle (e.g., [6]-[7] or [2] ). From this property and (3.10) we obtain

$$
\max _{x \in S_{R}} \Phi_{k}(x)=\max _{x \in \Omega_{R}} \Phi_{k}(x) \quad \forall R \in\left[R_{0 k}, R_{k}\right],
$$


where, as usual, $S_{R}=\left\{x \in \mathbb{R}^{2}:|x|=R\right\}$ is a circle and $\Omega_{R}=\Omega \cap B_{R}=\{x \in$ $\Omega:|x|<R\}$. From this maximum principle it follows that

the function $\left[R_{0 k}, R_{k}\right] \ni R \mapsto \max _{x \in S_{R}} \Phi_{k}(x) \quad$ is strictly increasing.

In particular, by (3.10),

$$
\max _{x \in S_{R}} \Phi_{k}(x)>\frac{1}{60} \quad \forall R \in\left[R_{0 k}, R_{k}\right] .
$$

STEP 4. By results of [6]-[7](see the proof of Lemma 4.1 in [6], in particular, the proof of the estimate (4.5)) there exist a sequence of radii $R_{m k}, m=$ $1,2, \ldots, M=M(k)$, such that

$$
\begin{array}{r}
2^{m-1} R_{0 k}<R_{m k}<2^{m} R_{0 k}, \quad m=1,2, \ldots, M ; \\
\frac{1}{3} R_{k}<R_{M k}<\frac{2}{3} R_{k} ; \\
\sup _{x \in S_{R_{m k}}}\left|\mathbf{u}_{k}(x)-\overline{\mathbf{u}}_{k}\left(R_{m k}\right)\right|=\varepsilon_{k} .
\end{array}
$$

Taking $k$ large enough and using estimates (3.14) for the Bernoulli pressure $\Phi_{k}$ and estimates (3.6) for the pressure $p_{k}$, we conclude from (3.17) that

$$
\left|\overline{\mathbf{u}}_{k}\left(R_{m k}\right)\right|=\left|f_{S_{R_{m k}}} \mathbf{u}_{k} d s\right|>\sqrt{2 \cdot \frac{1}{60}}+\varepsilon_{k}>\frac{1}{6} \quad \forall m=1,2, \ldots, M .
$$

Then, applying Lemma 2.1 and using the smallness of the Dirichlet integrals (3.1) and the condition (3.18), (3.15), we obtain

$$
\left|\overline{\mathbf{u}}_{k}(R)\right|=\left|f_{S_{R}} \mathbf{u}_{k} d s\right|>\frac{1}{7} \quad \forall R \in\left[R_{0 k}, R_{k}\right] .
$$

Denote by $\varphi_{k}(r)$ the angle direction of the vector $\overline{\mathbf{u}}_{k}(r)$, i.e.,

$$
\overline{\mathbf{u}}_{k}(r)=\left|\overline{\mathbf{u}}_{k}(r)\right|\left(\cos \varphi_{k}(r), \sin \varphi_{k}(r)\right) .
$$

From Corollary 2.1 and relations (3.1), (3.4), (3.16), (3.19) we have

$$
\sup _{R_{0 k}<\rho_{1} \leq \rho_{2} \leq R_{M k}}\left|\varphi_{k}\left(\rho_{2}\right)-\varphi_{k}\left(\rho_{1}\right)\right|=\varepsilon_{k} .
$$

Further, Lemma 2.1 and the boundary conditions $\left.\mathbf{u}_{k}(z)\right|_{|z|=R_{k}}=(1,0)$ yield

$$
\left|\overline{\mathbf{u}}_{k}(r)-(1,0)\right|=\varepsilon_{k} \quad \forall r \in\left[R_{M k}, R_{k}\right] .
$$


From the last two formulas we conclude that

$$
\sup _{R_{0 k} \leq r \leq R_{k}}\left|\varphi_{k}(r)\right|=\varepsilon_{k} .
$$

Summarizing we can say that the formula (3.20) holds with

$$
\left|\overline{\mathbf{u}}_{k}(r)\right| \geq \frac{1}{7}, \quad\left|\varphi_{k}(r)\right|=\varepsilon_{k} \quad \forall r \in\left[R_{0 k}, R_{k}\right] .
$$

STEP 5. From the choice of the radii $R_{1 k}$ and $R_{M k}$ at Step 4 (see (3.15)(3.16)), from estimates (3.6), (3.17), and from the conditions $\left|\overline{\mathbf{u}}_{k}\left(R_{0 k}\right)\right|=\frac{1}{5}$ (see (3.7)) and $\left|\overline{\mathbf{u}}_{k}\left(R_{k}\right)\right|=1$, we have

$$
\begin{aligned}
& \sup _{x \in S_{R_{1 k}}}\left|\Phi_{k}(x)-\frac{1}{50}\right| \rightarrow 0, \\
& \sup _{x \in S_{R_{M k}}}\left|\Phi_{k}(x)-\frac{1}{2}\right| \rightarrow 0
\end{aligned}
$$

as $k \rightarrow \infty$. Without loss of generality we may assume that

$$
\begin{array}{cl}
\Phi_{k}(x)<\frac{1}{45} & \forall x \in S_{R_{1 k}}, \\
\Phi_{k}(x)>\frac{1}{3} & \forall x \in S_{R_{M k}} .
\end{array}
$$

Denote by $I$ the interval $I=\left[\frac{1}{45}, \frac{1}{3}\right]$. By construction and by the classical Morse-Sard Theorem (which says that the set of critical values of a $C^{\infty}$ function has zero Lebesgue measure) we conclude that for almost all $t \in I$ the set

$$
\left\{x \in \mathbb{R}^{2}: R_{1 k} \leq|x| \leq R_{M k}, \quad \Phi_{k}(x)=t\right\}
$$

is a finite disjoint union of smooth closed curves. Moreover, every of these curves is homeomorphic to the circle. (It follows from the fact, that the preimage of a non-critical value is a smooth one dimensional manifold, and, since $\Phi_{k}(x) \notin I$ for $x \in S_{R_{1 k}}$ and $x \in S_{R_{M k}}$, this manifold has no boundary.) By evident topological reasons, at least one of these curves separate the circles $S_{R_{1 k}}$ and $S_{R_{M k}}$. By maximum principle for the Bernoulli pressure $\Phi_{k}$ this separating curve is unique; denote it by $S_{k}(t)$. In other words, we have proved that

$$
\begin{gathered}
\text { for almost all } t \in I=\left[\frac{1}{45}, \frac{1}{3}\right] \text { there exists exactly one smooth curve } \\
S_{k}(t) \text {, homeomorphic to the circle separating } S_{R_{1 k}} \text { from } S_{R_{M k}}, \\
\text { and satisfying the identity } \Phi_{k}(x) \equiv t \quad \forall x \in S_{k}(t) .
\end{gathered}
$$


STEP 6. Take numbers $t_{1} \in\left[\frac{1}{45}, \frac{1}{40}\right], t_{2} \in\left[\frac{1}{4}, \frac{1}{3}\right]$ which are regular values for all $\Phi_{k}, \quad k=1,2, \ldots$ Then denote by $\Omega_{k}^{s}$ the bounded open subset of $\Omega_{k}$ satisfying

$$
\partial \Omega_{k}^{s}=S_{k}\left(t_{1}\right) \cup S_{k}\left(t_{2}\right)
$$

We claim that

$$
\text { vorticity } \omega_{k}(x) \text { does not change sign in } \Omega_{k}^{s} \text {. }
$$

In order to prove this claim, consider the auxiliary function

$$
\gamma_{k}=\Phi_{k}-\omega_{k} \psi_{k}
$$

where $\psi_{k}$ is a stream function satisfying $\nabla \psi_{k}=\mathbf{u}_{k}^{\perp}=\left(-u_{k}^{2}, u_{k}^{1}\right)$ ( the function $\gamma_{k}$ was introduced by Amick in the paper [1]). By direct calculation,

$$
\nabla \gamma_{k}=-\nabla^{\perp} \omega_{k}-\psi_{k} \nabla \omega_{k}
$$

Then

$$
\nabla \gamma_{k} \cdot \nabla^{\perp} \omega_{k}=-\left|\nabla \omega_{k}\right|^{2}
$$

In other words,

$$
\frac{\partial \gamma_{k}}{\partial s}:=\nabla \gamma_{k} \cdot \frac{\nabla^{\perp} \omega_{k}}{\left|\nabla \omega_{k}\right|} \equiv-\left|\nabla \omega_{k}\right|,
$$

where we denote by $\frac{\partial \gamma_{k}}{\partial s}$ the derivative of $\gamma_{k}$ with respect to the direction tangent to the level set $\omega_{k}=c$. The last identities imply the following monotonicity properties

$\gamma_{k}$ is monotone along level sets of the vorticity $\omega_{k}=c$ and vice versa - the vorticity $\omega_{k}$ is monotone along level sets of $\gamma_{k}=c$

(see [1]). Moreover, there holds the evident identity

$$
\gamma_{k}=\Phi_{k} \quad \text { whenever } \quad \omega_{k}=0 .
$$

Suppose (3.30) is not true. Let $V_{k}$ be a connected component of the open set $\left\{x \in \Omega_{k}^{s}: \omega_{k}(x)>0\right\}$. By our assumption, $V_{k} \neq \Omega_{k}^{s}$, therefore, $\Omega_{k}^{s} \cap \partial V_{k} \neq \emptyset$. Take a decreasing sequence of noncritical values $\tau_{m}>0$ of the vorticity $\omega_{k}$ satisfying $\tau_{m} \rightarrow 0$ as $m \rightarrow \infty$. Since $\omega_{k}$ is a real analytical function in $\Omega_{k}$, for every $\tau_{m}$ the set

$$
\left\{z \in \partial \Omega_{k}^{s}: \omega_{k}(z)=\tau_{m}\right\}
$$


is finite. Using this fact and regularity of the values $\tau_{m}$, it is easy to see that the set

$$
\left\{z \in \bar{\Omega}_{k}^{s}: \omega_{k}(z)=\tau_{m}\right\}
$$

is a finite disjoint union of smooth curves homeomorphic to the unit interval $[0,1]$ with endpoints on $\partial \Omega_{k}^{s}$ (note, that $\tau_{m}$-level set can not contain curves homeomorphic to the circle because of the monotonicity property (3.33)).

Fix $z_{0} \in V_{k}$ and denote by $V_{k, m}$ the sequence of the connected components of the open set $\left\{x \in V_{k}: \omega_{k}(x)>\tau_{m}\right\}$ containing $z_{0}$. Evidently,

$$
V_{k, m} \subset V_{k}
$$

and

$$
V_{k}=\bigcup_{m \in \mathbb{N}} V_{k, m}
$$

Now we have to consider three possible cases:

(i) the equality

$$
S_{k}\left(t_{1}\right) \cap \partial V_{k, m}=\emptyset
$$

holds for all $m \in \mathbb{N}$;

(ii) the equality

$$
S_{k}\left(t_{2}\right) \cap \partial V_{k, m}=\emptyset
$$

holds for all $m \in \mathbb{N}$;

(iii) the relations

$$
S_{k}\left(t_{1}\right) \cap \partial V_{k, m} \neq \emptyset \quad \text { and } \quad S_{k}\left(t_{2}\right) \cap \partial V_{k, m} \neq \emptyset
$$

hold for all sufficiently large $m \geq m_{0}$.

Consider the case (i). First of all, we claim that for this case the relation

$$
S_{k}\left(t_{2}\right) \cap \partial V_{k, m} \neq \emptyset
$$

holds for all $m$. Indeed, if $S_{k}\left(t_{2}\right) \cap \partial V_{k, m}=\emptyset$, then from (3.37) we have $\partial V_{k, m} \cap\left(\partial \Omega_{k}^{s}\right)=\emptyset$, but this contradicts the strong maximum principle for the vorticity $\omega_{k}$ (see, e.g., [7]).

By (3.37), (3.40) there exists evidently a smooth $\operatorname{arc} L_{m} \subset V_{k} \cap \partial V_{k, m}$ such that

$$
\omega_{k} \equiv \tau_{m} \quad \text { on } L_{m},
$$




$$
L_{m} \text { separates the point } z_{0} \text { from the cycle } S_{k}\left(t_{1}\right) \text {, }
$$

the endpoints $A_{m}, B_{m}$ of the arc $L_{m}$ belong to the cycle $S_{k}\left(t_{2}\right)$.

(By cycle we mean the plane curve which is homeomorphic to the unit circle.) In particular, from the assertion (3.42) it follows, that

the $\operatorname{arc} L_{m}$ does not degenerate to a point when $m \rightarrow \infty$.

In other words,

$$
\operatorname{diam} L_{m} \nrightarrow 0 \quad \text { as } m \rightarrow \infty .
$$

By construction we have

$$
\begin{aligned}
\gamma\left(B_{m}\right)-\gamma\left(A_{m}\right) & =\Phi_{k}\left(B_{m}\right)-\Phi_{k}\left(A_{m}\right)-\tau_{m}\left(\psi\left(B_{m}\right)-\psi\left(A_{m}\right)\right) \\
& =-\tau_{m}\left(\psi\left(B_{m}\right)-\psi\left(A_{m}\right)\right) \rightarrow 0
\end{aligned}
$$

as $m \rightarrow \infty$. From this fact and from the properties (3.45), (3.32)-(3.33) (which could be applied because of (3.41)) it follows that

$$
\sup _{z \in L_{m}}\left|\nabla \omega_{k}(z)\right| \rightarrow 0 \quad \text { as } m \rightarrow \infty .
$$

But the last assertion, in view of (3.44)-(3.45), contradicts the fact that $\omega_{k}$ is a nonconstant real analytical function (in particular, $\nabla \omega_{k}$ can not be identically zero on a compact connected set which is not a single point).

The case (ii) can be proved exactly by the same arguments.

Consider the last possible case (iii). In this case evidently there exist two smooth $\operatorname{arcs} L_{m}^{+}$and $L_{m}^{-}$with the following properties

(o) the $\operatorname{arcs} L_{m}^{+}$and $L_{m}^{-}$are homeomorphic to the closed unit interval [0,1], their endpoints $A_{m}^{+}, B_{m}^{+}$and $A_{m}^{-}, B_{m}^{-}$satisfy

$$
A_{m}^{+}, A_{m}^{-} \in S_{k}\left(t_{1}\right) ; \quad B_{m}^{+}, B_{m}^{-} \in S_{k}\left(t_{2}\right) .
$$

(oo) the identity

$$
\omega_{m}(z) \equiv \tau_{m}
$$

holds for all $z \in L_{m}^{+}$and $L_{m}^{-}$;

(०००) the function $\gamma$ is increasing along $L_{m}^{+}$in the direction from $A_{m}^{+}$to $B_{m}^{+}$; and $\gamma$ is decreasing along $L_{m}^{-}$in the direction from $A_{m}^{-}$to $B_{m}^{-}$. 
From the last property it follows that

$$
\begin{gathered}
0 \leq \gamma\left(A_{m}^{-}\right)-\gamma\left(B_{m}^{-}\right)=\Phi_{k}\left(A_{m}^{-}\right)-\Phi_{k}\left(B_{m}^{-}\right)-\tau_{m}\left(\psi\left(A_{m}^{-}\right)-\psi\left(B_{m}^{-}\right)\right) \\
=\left(t_{1}-t_{2}\right)-\tau_{m}\left(\psi\left(B_{m}^{-}\right)-\psi\left(A_{m}^{-}\right)\right) .
\end{gathered}
$$

Since by construction $t_{2}>t_{1}$ and $\tau_{m} \rightarrow 0$ as $m \rightarrow \infty$, we conclude that the right hand side of the last formula is strictly negative for sufficiently large $m$, and that is a required contradiction.

Thus, the property (3.30) is proved. Without loss of generality we may assume that

$$
\omega_{k}(z)>0 \quad \forall z \in \Omega_{k}^{s} .
$$

STEP 7. For an angle $\theta \in(0,2 \pi)$ denote by $L_{\theta}$ the ray starting from the origin:

$$
L_{\theta}=\left\{s(\cos \theta, \sin \theta): s \in \mathbb{R}_{+}\right\} .
$$

Because of assumptions on smallness of the integrals (3.1), (3.4) there exists a value $\tilde{\theta}$ such that

$$
\left|\tilde{\theta}-\frac{3}{2} \pi\right|<\frac{1}{9},
$$

and

$$
\begin{gathered}
\int_{L_{\tilde{\theta}} \cap \Omega_{k}} r\left|\nabla \mathbf{u}_{k}\right|^{2}(r, \tilde{\theta}) d r \leq \varepsilon_{k}, \\
\int_{L_{\tilde{\theta}} \cap \Omega_{k}} r\left|\nabla \tilde{\mathbf{u}}_{k}\right|^{2}(r, \tilde{\theta}) d r \leq \varepsilon_{k}, \\
\int_{L_{\tilde{\theta}} \cap \Omega_{k}^{\prime}} r^{2}\left|\nabla \omega_{k}\right|^{2}(r, \tilde{\theta}) d r \leq \varepsilon_{k},
\end{gathered}
$$

where we denote $\tilde{\mathbf{u}}_{k}(r, \theta):=\mathbf{u}_{k}(r, \theta)-\overline{\mathbf{u}}_{k}(r)$ and as usual $\varepsilon_{k} \rightarrow 0$. By estimate (3.17),

$$
\sup _{m=1, \ldots, M}\left|\tilde{\mathbf{u}}_{k}\left(R_{m k}, \tilde{\theta}\right)\right| \leq \varepsilon_{k} .
$$

Therefore, the inequality (3.51) and the assumption (3.15) yield

$$
\sup _{R_{m 1} \leq r \leq R_{M k}}\left|\tilde{\mathbf{u}}_{k}(r, \tilde{\theta})\right| \leq \varepsilon_{k} .
$$

Then from the identities (3.20)-(3.23) we conclude, that

$$
\mathbf{u}_{k}(r, \tilde{\theta})=f_{k}(r)\left(\cos \varphi_{k}(r), \sin \varphi_{k}(r)\right),
$$


where

$$
f_{k}(r)=\left|\mathbf{u}_{k}(r, \tilde{\theta})\right|>0, \quad\left|\varphi_{k}(r)\right|=\varepsilon_{k} \quad \forall r \in\left[R_{m 1}, R_{M k}\right] .
$$

SteP 8. By construction, the considered domain $\Omega_{k}^{s}$ is contained in the ring

$$
R_{1 k} \leq|z| \leq R_{M k} \quad \forall z \in \Omega_{k}^{s}
$$

(see $(3.28))$.

Consider the intersection of the annulus-shape domain $\Omega_{k}^{s}$ with the ray $L_{\tilde{\theta}}$. Take a segment $[A, B] \subset L_{\tilde{\theta}}$ with endpoints $A, B$ satisfying

$$
\begin{gathered}
|A|=r_{1}<r_{2}=|B|, \\
A \in S_{k}\left(t_{1}\right), \quad B \in S_{k}\left(t_{2}\right),
\end{gathered}
$$

the interior of the segment $[A, B]$ is contained in the set $\Omega_{k}^{s} \cap L_{\tilde{\theta}}$

(the existence of such segment is geometrically evident since $\partial \Omega_{k}^{s}=S_{k}\left(t_{1}\right) \cup$ $S_{k}\left(t_{2}\right)$ and the cycle $S_{k}\left(t_{2}\right)$ surrounds the cycle $\left.S_{k}\left(t_{1}\right)\right)$.

Then, by construction and (1.16), we have

$$
\begin{aligned}
& 0<t_{2}-t_{1}=\Phi_{k}(B)-\Phi_{k}(A)=\int_{[A, B]} \nabla \Phi_{k} \cdot \mathbf{e}_{\tilde{\theta}} d r \\
= & -\int_{[A, B]} \nabla^{\perp} \omega_{k} \cdot \mathbf{e}_{\tilde{\theta}} d r+\int_{[A, B]} \omega_{k} \mathbf{u}_{k}^{\perp} \cdot \mathbf{e}_{\tilde{\theta}} d r=I+I I,
\end{aligned}
$$

where $\mathbf{e}_{\tilde{\theta}}=(\cos \tilde{\theta}, \sin \tilde{\theta})$. Estimate the terms $I$ and $I I$ separately:

$$
I \leq \sqrt{\int_{[A, B]} r^{2}\left|\nabla \omega_{k}\right|^{2} d r} \sqrt{\int_{[A, B]} \frac{1}{r^{2}} d r} \stackrel{(3.52)}{<} \varepsilon_{k} .
$$

By formulas (3.54)-(3.55), we have

$$
\mathbf{u}_{k}^{\perp}(r, \tilde{\theta})=f_{k}(r)\left(\cos \tilde{\varphi}_{k}(r), \sin \tilde{\varphi}_{k}(r)\right),
$$

where

$$
\tilde{\varphi}_{k}(r)=\frac{\pi}{2}-\varphi_{k}(r)
$$

Consequently,

$$
\frac{\pi}{2}-\varepsilon_{k}<\tilde{\varphi}_{k}(r)<\frac{\pi}{2}+\varepsilon_{k}
$$


By construction (see (3.49)), we also obtain

$$
\pi-\frac{1}{9}-\varepsilon_{k}<\tilde{\theta}-\tilde{\varphi}_{k}(r)<\pi+\frac{1}{9}+\varepsilon_{k} .
$$

Finally, we conclude

$$
\mathbf{u}_{k}^{\perp}(\tilde{\theta}, r) \cdot \mathbf{e}_{\tilde{\theta}}<0 \quad \forall r \in\left(R_{m 1}, R_{M k}\right) \supset[|A|,|B|] .
$$

Therefore, from (3.48) it follows that

$$
\omega_{k} \mathbf{u}_{k}^{\perp}(\tilde{\theta}, r) \cdot \mathbf{e}_{\tilde{\theta}}<0 \quad \forall r \in[|A|,|B|] .
$$

Consequently, the second term $I I$ in (3.60) is negative:

$$
I I<0
$$

and (3.60)-(3.61) imply the inequality

$$
t_{2}-t_{1}<\varepsilon_{k},
$$

contradicting the choice of $t_{2}, t_{1}$ (recall, that $t_{2}-t_{1} \geq \frac{1}{4}-\frac{1}{40}=\frac{9}{40}>\frac{1}{5}$, see Step 5). This contradiction finishes the proof of Theorem 1.1.

The proof of Theorem 1.2 is analogous. The only differences are the following. The pressure again is "almost zero", i.e., $\left|p_{k}\right|=\varepsilon_{k}$ in the subdomain $\Omega_{k}^{\prime}=B_{\frac{3}{4} R_{k}}$, but $\mathbf{u}_{k} \equiv(1,0)$ on $\partial \Omega$ and $\mathbf{u}_{k}$ is zero on the big circle $S_{R_{k}}$. Denote

$$
R_{0 k}=\max \left\{r \leq R_{k}:\left|f_{S_{r}} \mathbf{u}_{k} d s\right|=\frac{1}{5}\right\} .
$$

By the same reasons as above,

$$
R_{0 k} \rightarrow+\infty \quad \text { and } \quad \frac{R_{k}}{R_{0 k}} \rightarrow+\infty
$$

as $k \rightarrow \infty$. Then by the maximum principle for the Bernoulli pressure,

the function $\left[R_{0}, R_{0 k}\right] \ni R \mapsto \max _{x \in S_{R}} \Phi_{k}(x) \quad$ is strictly decreasing,

where $R_{0}$ is some fixed radius with $B_{R_{0}} \supset \partial \Omega\left(R_{0}\right.$ does not depend on $\left.k\right)$. Then Steps $3-8$ of the proof repeat exactly the corresponding steps in the proof of Theorem 1.1 with the following obvious change: now the Bernoulli pressure $\Phi_{k}$ is decreasing with respect to $R$, so that the circle-type level set $S_{k}\left(t_{1}\right) \subset\left\{\Phi_{k}=\right.$ $\left.t_{1}\right\}$ surrounds the level set $S_{k}\left(t_{2}\right) \subset\left\{\Phi_{k}=t_{2}\right\}$ for $t_{1}<t_{2}$. 


\section{References}

[1] C.J. Amick: On Leray's problem of steady Navier-Stokes flow past a body in the plane, Acta Math 161 (1988), 71-130. 2, 3, 6, 9, 13

[2] L.C. Evans: Partial differential equations, AMS Graduate Studies in Mathematics, Vol. 19, 2-nd edition, (2010). 10

[3] R. Finn And D.R. Smith: On the stationary solutions of the NavierStokes equations in two dimensions, Arch. Ration. Mech. Anal. 25 (1967), $26-39.5$

[4] H. Fujita: On the Existence and Regularity of the Steady-State Solutions of the Navier-Stokes Equation, J. Fac. Sci. Univ. Tokyo(1A) 9 (1961), 59102. 5

[5] G.P. Galdi: An Introduction to the Mathematical Theory of the NavierStokes Equations. Steady-state problems, Springer, (2011). 2, 3, 5, 9

[6] D. Gilbarg And H.F. Weinberger: Asymptotic properties of Leray's solution of the stationary two-dimensional Navier-Stokes equations, Russian Math. Surveys 29 (1974), 109-123. 3, 6, 9, 10, 11

[7] D. Gilbarg and H.F. Weinberger: Asymptotic properties of steady plane solutions of the Navier-Stokes equations with bounded Dirichlet integral, Ann. Scuola Norm. Super. Pisa (4) 5 (1978), 381-404. 6, 8, 9, $10,11,14$

[8] M.V. Korobkov, K. Pileckas and R. Russo: The existence of a solution with finite Dirichlet integral for the steady Navier-Stokes equations in a plane exterior symmetric domain, J. Math. Pures. Appl. 101 (2014), $257-274.5$

[9] M.V. Korobkov, K. Pileckas and R. Russo, On the steady NavierStokes equations in 2D exterior domains, http://arxiv.org/abs/1711.02400 5

[10] M.V. Korobkov, K. Pileckas and R. Russo, On convergence of arbitrary D-solution of steady Navier-Stokes system in $2 \mathrm{D}$ exterior domains, Arch. Rational Mech. Anal. (Online first, 2019), https://doi.org/10.1007/s00205-019-01359-8 5

[11] O.A. Ladyzhenskaia: The Mathematical theory of viscous incompressible fluid, Gordon and Breach (1969). 3 
[12] J. Leray: Étude de diverses équations intégrales non linéaire et de quelques problèmes que pose l'hydrodynamique, J. Math. Pures Appl. 12 (1933), 1-82. 2

[13] K. Pileckas and R. Russo: On the existence of vanishing at infinity symmetric solutions to the plane stationary exterior Navier-Stokes problem, Math. Ann. 352 (2012), 643-658. 5

[14] A. Russo: A note on the two-dimensional steady-state Navier-Stokes problem, J. Math. Fluid Mech., 11 (2009) 407-414. 5

[15] R. Russo: On Stokes' problem, in Advances in Mathematica Fluid Mechanics, Eds. R. Rannacher and A. Sequeira, p. 473-511, Springer-Verlag Berlin Heidelberg (2010).

3 